\title{
COMPARISON THEOREMS FOR CAUSAL FUNCTIONAL DIFFERENTIAL EQUATIONS
}

\author{
ALEX MCNABB AND GRAHAM WEIR
}

(Communicated by Kenneth R. Meyer)

\begin{abstract}
Weak and strong comparison theorems are proved for causal functional differential equations
\end{abstract}

Introduction. In [1], comparison theorems were derived for initial value problems associated with ordinary differential equations. In this note, the ideas and techniques are applied to causal functional differential equations of the form

$$
\frac{d}{d t} y(t)=f(t, y), \quad t \in(a, b),
$$

where $y \in S_{n}$ the space of continuous maps from $[a, b] \subset R$ into $R^{n}$, which are differentiable on $(a, b)$, and $f$ is a map from $[a, b] \times S_{n}$ into $R^{n}$, which is causal in the sense that

$$
f(t, u)=f(t, v), \quad t \in[a, b]
$$

for all $u, v \in S_{n}$ such that $u(s)=v(s)$ when $a \leq s \leq t$.

If $y, z \in S_{n}$ we may define the functions $\bar{f}(t, y, z), \underline{f}(t, y, z)$ mapping $[a, b] \times S_{n}^{2}$ into $R^{n}$ as follows; componentwise,

$$
\bar{f}(t, y, z)=\sup f(t, \theta), \quad \underline{f}(t, y, z)=\inf f(t, \theta),
$$

where $\theta \in S_{n}$ and for every component $i$, and each point $s$ in $[a, b]$,

$$
\min \left(y_{i}(s), z_{i}(s)\right) \leq \theta_{i}(s) \leq \max \left(y_{i}(s), z_{i}(s)\right) .
$$

1 .

WEAK COMPARISON THEOREM Suppose $\underline{y}, y, \bar{y} \in S_{n}, f(t, y)$ is causal and

$$
\underline{y}(a)<y(a)<\bar{y}(a)
$$

$$
\frac{d}{d t} \underline{y}(t)-\underline{f}(t, \underline{y}, \bar{y})<\frac{d}{d t} y(t)-f(t, y)<\frac{d}{d t} \bar{y}(t)-\bar{f}(t, \underline{y}, \bar{y}) \quad \text { on }(a, b] .
$$

Then

$$
\underline{y}(t)<y(t)<\bar{y}(t) \quad \text { on }[a, b] .
$$

PROOF. If the inequality (3) does not hold, there is a point $x$ in $(a, b]$ such that (3) holds in $[a, x)$, but $y_{i}(x)=\bar{y}_{i}(x)$ or $\underline{y}_{i}(x)$ for some component $y_{i}$ of $y$. Suppose $y_{i}(x)=\bar{y}_{i}(x)$.

Received by the editors April 10, 1987 and, in revised form, September 21, 1987.

1980 Mathematics Subject Classification (1985 Revision). Primary 39C05, 34K15; Secondary $42 \mathrm{~B} 25$. 
Then

$$
\frac{d}{d t} y_{i}(x) \geq \frac{d}{d t} \bar{y}_{i}(x)
$$

and hence

$$
\frac{d}{d t} y_{i}(x)-f_{i}(x, y) \geq \frac{d}{d t} \bar{y}_{i}(x)-\bar{f}_{i}(x, \underline{y}, \bar{y}),
$$

since $\bar{f}_{i}(x, \underline{y}, \bar{y})=\sup f_{i}(x, \theta)$ for $\underline{y} \leq \theta \leq \bar{y}$ and $y$ is a candidate in the search for the supremum since $f$ is causal. This violates assumption (2) and a similar argument makes the alternative assumption $y_{i}(x)=\underline{y}_{i}(x)$ likewise untenable.

2.

STRONG COMPARISON THEOREM The weak theorem can be made stronger if $f(t, u)$ satisfies a Lipschitz condition of the form

$$
\begin{aligned}
\|f(t, u)-f(t, v)\| & =\sup _{i}\left|f_{i}(t, u)-f_{i}(t, v)\right| \leq K\|u-v\|_{t} \\
& =K \sup \left\{\left|u_{j}(s)-v_{j}(s)\right| \mid 1 \leq j \leq n, s \in(a, t)\right\} .
\end{aligned}
$$

Suppose $y, \bar{y}, y \in S_{n} ; f(t, y)$ is a causal locally Lipschitz continuous map in the sense described above, and

$$
\begin{gathered}
\underline{y}(a)<y(a)<\bar{y}(a) \\
\frac{d}{d t} \underline{y}(t)-\underline{f}(t, \underline{y}, \bar{y}) \leq \frac{d}{d t} y-f(t, y) \leq \frac{d}{d t} \bar{y}-\bar{f}(t, \underline{y}, \bar{y}) .
\end{gathered}
$$

Then

$$
\underline{y}<y<\bar{y} \text { on }[a, b] .
$$

ProOF. Let $w \in R^{n}$ be such that $w>0$ and $2 w<\bar{y}(a)-y(a)$ and $y(a)-\underline{y}(a)$ componentwise. Define $\bar{u}=\bar{y}-e^{-2 K(t-a)} w, \underline{u}=\underline{y}+e^{-2 K(t-a)} w$, and note that $\underline{y}(a)<\bar{u}(a)<y(a)<\bar{u}(a)<\bar{y}(a)$.

Since $\bar{u}, \underline{u}$ are continuous on $[a, b]$ and $\bar{u}(a)>\underline{u}(a)$, there is an $x$ in $[a, b]$ such that $[a, x]$ is the maximal interval for which $\underline{u}(s) \leq y(s) \leq \bar{u}(s)$ for all $s$ in $[a, x]$. Consider the expression $\bar{E}$ defined by

$$
\begin{aligned}
\bar{E} & =\frac{d}{d t} \bar{u}-\bar{f}(t, \underline{u}, \bar{u}) \\
& =\left\{\frac{d}{d t} \bar{y}-\bar{f}(t, \underline{y}, \bar{y})\right\}+2 K e^{-2 K(t-a)} w+\{\bar{f}(t, \underline{y}, \bar{y})-\bar{f}(t, \underline{u}, \bar{u})\} \\
& \geq\left\{\frac{d}{d t} y-f(t, y)\right\}+2 K e^{-2 K(t-a)} w+\{\bar{f}(t, \underline{y}, \bar{y})-\bar{f}(t, \underline{u}, \bar{u})\} .
\end{aligned}
$$

By definition,

$$
\begin{aligned}
& \bar{f}_{i}(t, \underline{y}, \bar{y})=\sup f_{i}(t, \theta) \\
& \quad \text { for }\left\{\theta: \theta \in S_{n} \text { and } \min (\underline{y}(s), \bar{y}(s)) \leq \theta(s) \leq \max (\underline{y}(s), \bar{y}(s))\right\} \\
& \bar{f}_{i}(t, \underline{u}, \bar{u})=\sup f_{i}(t, \theta) \\
& \quad \text { for }\left\{\theta: \theta \in S_{n} \text { and } \min (\underline{u}(s), \bar{u}(s)) \leq \theta(s) \leq \max (\underline{u}(s), \bar{u}(s))\right\} .
\end{aligned}
$$


On the closed interval $[a, x], \bar{f}_{i}(t, \underline{u}, \bar{u}) \leq \bar{f}_{i}(t, \underline{y}, \bar{y})$, and hence

$$
\bar{E}>\frac{d}{d t} y-f(t, y) \quad \text { on }[a, x] \text {. }
$$

In similar fashion

$$
\underline{E}=\frac{d}{d t} \underline{u}-\underline{f}(t, \underline{u}, \bar{u})<\frac{d}{d t} y-f(t, y) \quad \text { on }[a, x] .
$$

By the weak comparison theorem $\underline{u}<y<\bar{u}$ on $[a, x]$ and since $[a, x]$ is maximal, we must conclude $x=b$, and $\underline{y}<\underline{u}<y<\bar{u}<\bar{y}$ on $[a, b]$.

3. An example. Consider the differential delay equation

$$
\begin{aligned}
\frac{d}{d t} y(t) & =A(t) y(t-h), \quad t \in(h, b), 0<h<b, \\
& =A(t) g(t), \quad t \in(0, h], y(0)=g(h),
\end{aligned}
$$

where $y \in S_{n}$, and $A(t)$ is an $n \times n$ matrix. For this example,

$$
f(t, y) \equiv A(t) y(t-h) \quad \text { when } t \in(h, b)
$$

and

$$
\begin{aligned}
\bar{f}_{i}(t, \underline{y}, \bar{y}) & \equiv \sup _{\underline{y} \leq \theta \leq \bar{y}} \sum_{j=1}^{n} A(t)_{i j} \theta_{j}(t-h) \\
& =\left[A^{+}(t) \bar{y}(t-h)+A^{-}(t) \underline{y}(t-h)\right]_{i},
\end{aligned}
$$

where $A^{+}(t)$ is the matrix with $A^{+}(t)_{i j}=A(t)_{i j}$ if $A(t)_{i j} \geq 0$ and $A^{+}(t)_{i j}=0$ otherwise. $A^{-}(t)$ similarly is the matrix of negative elements of $A(t)$ and evidently $A(t) \equiv A^{+}(t)+A^{-}(t)$. Likewise,

$$
\underline{f}(t, \underline{y}, \bar{y})=A^{+}(t) \underline{y}(t-h)+A^{-}(t) \bar{y}(t-h) .
$$

We are led to associate the $2 n$ order system

$$
\begin{aligned}
\frac{d}{d t} \bar{y} & \geq A^{+}(t) \bar{y}(t-h)+A^{-}(t) \underline{y}(t-h), \quad t \in(h, b), \\
\frac{d}{d t} \underline{y} & \leq A^{+}(t) \underline{y}(t-h)+A^{-}(t) \bar{y}(t-h), \\
\frac{d}{d t} \bar{y} & \geq A^{+}(t) \bar{g}(t)+A^{-}(t) \underline{g}(t), \quad t \in(0, h], \\
\frac{d}{d t} \underline{y} & \leq A^{+}(t) \underline{g}(t)+A^{-}(t) \bar{g}(t), \quad \bar{g}(s) \geq g(s) \geq \underline{g}(s), \quad s \in(0, h], \\
\bar{y}(0) & \geq \bar{g}(h), \quad \underline{y}(0) \leq \underline{g}(h),
\end{aligned}
$$

with system (3.1).

If $z(t)=\bar{y}(t)-\underline{y}(t)$, we see that

$$
\begin{aligned}
\frac{d}{d t} z & \geq\left(A^{+}(t)-A^{-}(t)\right) z(t-h) \quad \text { for } t \in(h, b), \\
\frac{d}{d t} z & \geq\left(A^{+}(t)-A^{-}(t)\right)(\bar{g}(t)-\underline{g}(t)) \quad \text { for } t \in(0, h] \\
z(0) & \geq \bar{g}(h)-\underline{g}(h) \geq 0 .
\end{aligned}
$$


One half of the strong comparison theorem applied to (3.3) shows that $z \geq 0$ on $(0, b)$ and $(d / d z) z \geq 0$ and $(0, b)$ and so, if

$$
\frac{d}{d t} \bar{z}(t)=|A(t)| \bar{z}(t)
$$

where $|A| \equiv A^{+}-A^{-}$, then

$$
\begin{aligned}
\frac{d}{d t} \bar{z}(t) & =|A(t)| \bar{z}(t-h)+|A(t)|(\bar{z}(t)-\bar{z}(t-h)) \\
& >|A(t)| \bar{z}(t-h) .
\end{aligned}
$$

Thus the differential equation (3.4) gives rise to upper bounds on $z(t)$.

\section{REFERENCES}

1. A. McNabb, Comparison theorems for differential equations, J. Math. Anal. Appl. 119 (1986), 417-428.

Department of SCientific and Industrial Research, Applied Mathematics Division, WELLINGTON, NEW ZEALAND 